\title{
Branched Polymers and Hyperplane Arrangements
}

\author{
Karola Mészáros • Alexander Postnikov
}

Received: 9 December 2011 / Revised: 7 November 2012 / Accepted: 25 March 2013 /

Published online: 23 April 2013

(C) Springer Science+Business Media New York 2013

\begin{abstract}
We generalize the construction of connected branched polymers and the notion of the volume of the space of connected branched polymers studied by Brydges and Imbrie (Ann Math, 158:1019-1039, 2003), and Kenyon and Winkler (Am Math Mon, 116(7):612-628, 2009) to any central hyperplane arrangement $\mathcal{A}$. The volume of the resulting configuration space of connected branched polymers associated to the hyperplane arrangement $\mathcal{A}$ is expressed through the value of the characteristic polynomial of $\mathcal{A}$ at 0 . We give a more general definition of the space of branched polymers, where we do not require connectivity, and introduce the notion of $q$-volume for it, which is expressed through the value of the characteristic polynomial of $\mathcal{A}$ at $-q$. Finally, we relate the volume of the space of branched polymers to broken circuits and show that the cohomology ring of the space of branched polymers is isomorphic to the Orlik-Solomon algebra.
\end{abstract}

Keywords Polymers · Braid arrangement · Hyperplane arrangement · Characteristic polynomial $\cdot$ Broken circuit $\cdot$ Orlik-Solomon algebra

\section{Introduction}

Brydges and Imbrie [2], and Kenyon and Winkler [3] study the space of branched polymers of order $n$ in $\mathbb{R}^{2}$ and $\mathbb{R}^{3}$. In their definition, a branched polymer of order $n$ in

K. Mészáros $(\varangle)$

Department of Mathematics, Cornell University, Ithaca, NY 14853, USA

e-mail:karola@math.cornell.edu

A. Postnikov

Department of Mathematics, Massachusetts Institute of Technology,

Cambridge, MA 02139, USA

e-mail: apost@math.mit.edu 
$\mathbb{R}^{D}$ is a connected set of $n$ labeled unit spheres in $\mathbb{R}^{D}$ with nonoverlapping interiors. To each polymer $P$ corresponds a graph $G_{P}$ on the vertex set $[n]$ where $(i, j) \in E\left(G_{P}\right)$ if and only if spheres $i$ and $j$ touch in the polymer. The space of branched polymers of order $n$ in $\mathbb{R}^{D}$ can be parametrized by the $D$-dimensional angles at which the spheres are attached to each other. If $G_{P}$ has a cycle, this parametrization becomes ambiguous. However, since polymers containing a cycle of touching spheres have probability zero, this ambiguity is not of concern. Brydges and Imbrie [2], and Kenyon and Winkler [3] compute the volume of the space of branched polymers in $\mathbb{R}^{2}$ and $\mathbb{R}^{3}$, which are $(n-1) !(2 \pi)^{n-1}$ and $n^{n-1}(2 \pi)^{n-1}$, respectively. They show that if we relax the requirement that the spheres in the polymer have radii 1 , the volume of the space of branched polymers in $\mathbb{R}^{2}$ remains the same, while the volume of the space of branched polymers in $\mathbb{R}^{3}$ changes.

Intrigued by the robustness of the space of branched polymers in $\mathbb{R}^{2}$ under the change of radii, we generalize this notion differently from how it is done in [2,3]. Under our notion of polymers, the volume of the space of polymers is independent of the radii in all cases.

We associate the space of branched polymers to any central hyperplane arrangement $\mathcal{A}$. The polymers corresponding to the braid arrangement $\mathcal{B}_{n}$ coincide with branched polymers in $\mathbb{R}^{2}$ as defined above. We also broaden the notions of branched polymers, by not requiring polymers to be connected. We define the volume of the space of such polymers and show that it is invariant under the change of radii. In the case of the braid arrangement, the volume of the space of connected branched polymers associated to $\mathcal{B}_{n}$ is $(n-1) !(2 \pi)^{n-1}=(-2 \pi)^{r\left(\mathcal{B}_{n}\right)} \chi_{\mathcal{B}_{n}}(0)$, where $r\left(\mathcal{B}_{n}\right)$ is the rank of $\mathcal{B}_{n}$ and $\chi_{\mathcal{B}_{n}}(t)$ is the characteristic polynomial of $\mathcal{B}_{n}$. In our generalized notion of volume, weighted with $q$, the volume of the space of branched polymers associated to $\mathcal{B}_{n}$ is $(-2 \pi)^{r\left(\mathcal{B}_{n}\right)} \chi_{\mathcal{B}_{n}}(-q)$. The volume of the space of connected branched polymers associated to $\mathcal{B}_{n}$ is a specialization of this $q$-volume at $q=0$.

A theorem of the same flavor holds for any of our polymers.

Theorem The q-volume of the space of branched polymers associated to a central hyperplane arrangement $\mathcal{A}$ is $(-2 \pi)^{r(\mathcal{A})} \chi_{\mathcal{A}}(-q)$. Furthermore, the volume of the space of connected branched polymers associated to $\mathcal{A}$ is a specialization of its $q$-volume at $q=0$.

In the case that $\mathcal{A}$ is a graphical arrangement, we recover the $G$-polymers of [3], and the Theorem can be rephrased in terms of the chromatic polynomial of the graph $G$. We also relate the volume of the space of branched polymers to broken circuits and the Orlik-Solomon algebra.

The outline of the paper is as follows. In Sect. 2 we explain how the notion of branched polymers in $\mathbb{R}^{2}$ from [2,3] translates to polymers associated to the braid arrangement $\mathcal{B}_{n}$. In Sect. 3 we give a general definition of connected branched polymers, as well as branched polymers (where we do not require connectivity) associated to any arrangement $\mathcal{A}$. We define the notion of $q$-volume of the space of branched polymers and restate the Theorem about the value of the $q$-volume of the space of branched polymers. In Sect. 4 we prove the Theorem. In Sect. 5 we recover the $G$-polymers of [3] from the graphical arrangement $\mathcal{A}_{G}$. We relate the volumes of branched polymers 
to broken circuits in Sect. 7. We conclude in Sect. 8 by proving that the cohomology ring of the space of branched polymers is the Orlik-Solomon algebra.

\section{Branched Polymers and the Braid Arrangement}

In this section we explain how to think of the space of connected branched polymers and its volume defined in [2,3] in terms of the braid arrangement $\mathcal{B}_{n}$. We also give a more general definition of the space of branched polymers associated to $\mathcal{B}_{n}$ equipped with the notion of $q$-volume. For $q=0$ we recover the volume of the space of connected branched polymers in the sense of $[2,3]{ }^{1}$

Let $V:=\mathbb{C}^{n} /(1, \ldots, 1) \mathbb{C} \cong \mathbb{C}^{n-1}$. A point $\mathbf{x} \in V$ has coordinates $\left(x_{1}, \ldots, x_{n}\right)$ considered modulo simultaneous shifts of all $x_{i}$ 's.

The braid arrangement $\mathcal{B}_{n}$ in $V, n,>1$ consists of the $\left(\begin{array}{l}n \\ 2\end{array}\right)$ hyperplanes given by the equations

$$
h_{i j}(\mathbf{x})=x_{i}-x_{j}=0 \text {, for } 1 \leq i<j \leq n, \text { where } \mathbf{x} \in V \text {. }
$$

Let

$$
H_{i j}=\left\{\mathbf{x} \in V \mid h_{i j}(\mathbf{x})=0\right\}
$$

Define the space of branched polymers associated to the arrangement $\mathcal{B}_{n}$ and positive scalars $R_{i j}, 1 \leq i<j \leq n$ to be

$$
P_{\mathcal{B}_{n}}=\left\{\mathbf{x} \in V|| h_{i j}(\mathbf{x}) \mid \geq R_{i j} \text { for all } H_{i j} \in \mathcal{B}_{n}\right\}
$$

A connected branched polymer of size $n$ is a connected collection of $n$ labeled disks in $\mathbb{R}^{2}$ with nonoverlapping interiors (considered up to translation). For an example of a connected branched polymer see Fig. 1 . Think of the collection of $n$ disks in $\mathbb{R}^{2}=\mathbb{C}$ as a point $\mathbf{x} \in V$, where $x_{k}$ is the center of the $k$ th disk. Denote by $r_{k}$ the radius of the $k$ th disk and let $R_{i j}=r_{i}+r_{j}$. The condition that the disks do not overlap can be written as $\left|x_{i}-x_{j}\right| \geq R_{i j}$. Disks $i$ and $j$ touch exactly if $\left|x_{i}-x_{j}\right|=R_{i j}$. Thus the space $P_{\mathcal{B}_{n}}$ consists of points corresponding to branched polymers, which are not necessarily connected.

It is clear from the definition of $P_{\mathcal{B}_{n}}$ that it decomposes as the disjoint union

$$
P_{\mathcal{B}_{n}}=\bigsqcup_{S \subset \mathcal{B}_{n}} P_{\mathcal{B}_{n}}^{S},
$$

where $P_{\mathcal{B}_{n}}^{S}$ consists of all points $\mathbf{x} \in P_{\mathcal{B}_{n}}$ such that $\left|x_{i}-x_{j}\right|=R_{i j}$ exactly if $H_{i j} \in S$. That is,

$$
P_{\mathcal{B}_{n}}^{S}=\left\{\mathbf{x} \in \mathbb{C}^{n}|| h_{i j}(\mathbf{x}) \mid>R_{i j} \text { for } H_{i j} \in \mathcal{B}_{n} \backslash S,\left|h_{i j}(\mathbf{x})\right|=R_{i j} \text { for } H_{i j} \in S\right\}
$$

\footnotetext{
1 Note that our terminology differs slightly from that of [2,3]. The notion of "branched polymer" in [2,3] corresponds to our "connected branched polymers."
} 
Fig. 1 A connected branched polymer

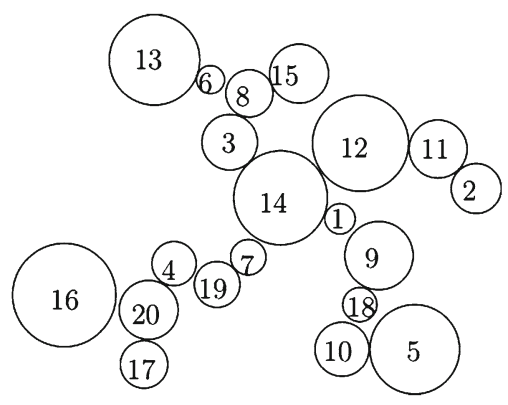

We can consider $\mathcal{B}_{n}$ as a matroid with ground set the set of hyperplanes in $\mathcal{B}_{n}$ and independence given by linear independence of the normals to the hyperplanes.

Connected branched polymers, in the sense of [2,3], correspond to points in the strata $P_{\mathcal{B}_{n}}^{S}$ of $P_{\mathcal{B}_{n}}$ such that $S$ contains a base of $\mathcal{B}_{n}$. Otherwise, the configuration of disks would not be connected. Thus, the space of connected branched polymers corresponding to $\mathcal{B}_{n}$ is

$$
C P_{\mathcal{B}_{n}}=\bigsqcup_{S \subset \mathcal{B}_{n}: r(S)=r\left(\mathcal{B}_{n}\right)} P_{\mathcal{B}_{n}}^{S},
$$

where $r(S)$ denotes the rank of the arrangement $S$, which is the dimension of the space which the normals to the hyperplanes in $S$ span.

We now define the notion of the volume of the space $C P_{\mathcal{B}_{n}}$, which coincides with the definition given in $[2,3]$.

Let $S=\left\{h_{i_{1} j_{1}}, \ldots, h_{i_{n-1} j_{n-1}}\right\}$ be a base of $\mathcal{B}_{n}$. Let us embed $P_{\mathcal{B}_{n}}^{S}$ into $[0,2 \pi]^{n-1}$ by

$$
\mathbf{x} \mapsto \varphi(\mathbf{x})=\left(\varphi_{1}, \ldots, \varphi_{n-1}\right),
$$

where $h_{i_{k} j_{k}}(\mathbf{x})=e^{i \varphi_{k}} R_{i_{k} j_{k}}, k \in[n-1]$. Define the volume of $P_{\mathcal{B}_{n}}^{S}$ as the volume of its image in $[0,2 \pi]^{n-1}$ :

$$
\operatorname{vol} P_{\mathcal{B}_{n}}^{S}=\operatorname{vol}\left(\left\{\varphi(\mathbf{x}) \mid \mathbf{x} \in P_{\mathcal{B}_{n}}^{S}\right\}\right)
$$

If $S$ is a dependent set in $\mathcal{B}_{n}$, then $P_{\mathcal{B}_{n}}^{S}$ has a lower dimension $\left(\operatorname{dim} P_{\mathcal{B}_{n}}^{S}<n-1\right)$, so we let vol $P_{\mathcal{B}_{n}}^{S}=0$.

Let

$$
\operatorname{vol} C P_{\mathcal{B}_{n}}=\sum_{S: r(S)=r\left(\mathcal{B}_{n}\right)} \operatorname{vol} P_{\mathcal{B}_{n}}^{S}=\sum_{S: S \in \mathcal{B}\left(\mathcal{B}_{n}\right)} \operatorname{vol} P_{\mathcal{B}_{n}}^{S}
$$

where $\mathcal{B}(\mathcal{M})$ denotes the set of bases of a matroid $\mathcal{M}$.

Recall that the characteristic polynomial of $\mathcal{B}_{n}$ is $\chi_{\mathcal{B}_{n}}(t)=(t-1) \cdots(t-(n-1))$ [5, p. 414]. By [3, Theorem 2], vol $C P_{\mathcal{B}_{n}}=(n-1) !(2 \pi)^{n-1}$. Observe that it equals 


$$
\operatorname{vol} C P_{\mathcal{B}_{n}}=(-2 \pi)^{r\left(\mathcal{B}_{n}\right)} \chi_{\mathcal{B}_{n}}(0)
$$

where $r\left(\mathcal{B}_{n}\right)=n-1$ is the rank of $\mathcal{B}_{n}$. Equation (1) is a special case of Theorem 1 which we state and prove in the following sections.

\section{Polymers Associated to a Hyperplane Arrangement}

In this section we associate branched polymers to any central hyperplane arrangement. We calculate the volume of the space of connected branched polymers as well as the $q$-volume of the space of all branched polymers.

Let $\left\{h_{1}, \ldots, h_{N}\right\} \subset\left(\mathbb{C}^{r}\right)^{*}$, and assume that $h_{1}, \ldots, h_{N}$ span $\left(\mathbb{C}^{r}\right)^{*}$. Let $\mathcal{A}=$ $\left\{H_{1}, \ldots, H_{N}\right\}$ be the hyperplane arrangement where the hyperplanes are

$$
H_{i}=\left\{\mathbf{x} \in \mathbb{C}^{r} \mid h_{i}(\mathbf{x})=0\right\}
$$

Note that $r=r(\mathcal{A})$ is the rank of the arrangement.

Define the space of branched polymers associated to the arrangement $\mathcal{A}$ and positive scalars $R_{i}, 1 \leq i \leq N$ to be

$$
P_{\mathcal{A}}=\left\{\mathbf{x} \in \mathbb{C}^{r}|| h_{i}(\mathbf{x}) \mid \geq R_{i} \text { for all } H_{i} \in \mathcal{A}\right\}
$$

This space can be thought of as $\mathbb{C}^{r}$ with $N$ tubes removed. Namely, let

$$
T_{i}=\left\{\mathbf{x} \in \mathbb{C}^{r}|| h_{i}(\mathbf{x}) \mid<R_{i}\right\}, i \in[N]
$$

be the $i$ th tube, which is the set of points in $\mathbb{C}^{r}$ at distance less than $R_{i} /\left\|h_{i}\right\|$ from the hyperplane $H_{i}$. Clearly then $P_{\mathcal{A}}$ is the complement to all the tubes in $\mathbb{C}^{r}$. The space $P_{\mathcal{A}}$ is related to the well-studied space $C_{\mathcal{A}}=\mathbb{C}^{r} \backslash \bigcup_{H \in \mathcal{A}} H$, the complement of the arrangement $\mathcal{A}$ in $\mathbb{C}^{r}$.

The space of branched polymers $P_{\mathcal{A}}$ decomposes as the disjoint union

$$
P_{\mathcal{A}}=\bigsqcup_{S \subset \mathcal{A}} P_{\mathcal{A}}^{S},
$$

where

$$
P_{\mathcal{A}}^{S}=\left\{\mathbf{x} \in \mathbb{C}^{r}|| h_{i}(\mathbf{x}) \mid>R_{i} \text { for } H_{i} \in \mathcal{A} \backslash S,\left|h_{i}(\mathbf{x})\right|=R_{i} \text { for } H_{i} \in S\right\} .
$$

Consider $\mathcal{A}$ as the matroid with ground set the hyperplanes in $\mathcal{A}$ and independence linear independence of the normals to the hyperplanes.

Define the space of connected branched polymers associated to $\mathcal{A}$ as

$$
C P_{\mathcal{A}}=\bigsqcup_{S \subset \mathcal{A}: r(S)=r(\mathcal{A})} P_{\mathcal{A}}^{S}
$$


We now define the notions of volume vol and $q$-volume $\operatorname{vol}_{q}$ of $P_{\mathcal{A}}^{S}$ for any set $S \subset \mathcal{A}$ with respect to $\mathcal{A}$. Let vol $P_{\mathcal{A}}^{S}=0$ for any dependent set $S$, since $\operatorname{dim} P_{\mathcal{A}}^{S}<n$.

Let $S=\left\{h_{i_{1}}, \ldots, h_{i_{r}}\right\}$ be a base of $\mathcal{A}$. Embed $P_{\mathcal{A}}^{S}$ into $[0,2 \pi]^{r}$ by

$$
\mathbf{x} \mapsto \varphi(\mathbf{x})=\left(\varphi_{1}, \ldots, \varphi_{r}\right),
$$

where $h_{i_{k}}(\mathbf{x})=e^{i \varphi_{k}} R_{i_{k}}, k \in[r]$. Define the volume of $P_{\mathcal{A}}^{S}$ as the volume of its image in $[0,2 \pi]^{r}$ :

$$
\operatorname{vol} P_{\mathcal{A}}^{S}=\operatorname{vol}\left(\left\{\varphi(\mathbf{x}) \mid \mathbf{x} \in P_{\mathcal{A}}^{S}\right\}\right)
$$

For any subset $S=\left\{H_{i_{1}}, \ldots, H_{i_{l}}\right\}$ of $\mathcal{A}$, let

$$
\langle S\rangle_{\mathcal{A}}:=\left\{H_{i} \in \mathcal{A} \mid h_{i} \text { is in the } \operatorname{span}\left\langle h_{i_{1}}, \ldots, h_{i_{l}}\right\rangle\right\} .
$$

We consider $\langle S\rangle_{\mathcal{A}}$ as a hyperplane arrangement in $\mathbb{C}^{r} /\left(H_{i_{1}} \cap \cdots \cap H_{i_{l}}\right) \cong \mathbb{C}^{r^{\prime}}$. Its rank is $r\left(\langle S\rangle_{\mathcal{A}}\right)=r^{\prime}$.

Define

$$
\operatorname{vol} P_{\mathcal{A}}^{S}:=\operatorname{vol} P_{\langle S\rangle_{\mathcal{A}}}^{S} \cdot(2 \pi)^{r(\mathcal{A})-r\left(\langle S\rangle_{\mathcal{A}}\right)},
$$

and

$$
\operatorname{vol}_{q} P_{\mathcal{A}}^{S}:=\operatorname{vol} P_{\mathcal{A}}^{S} \cdot q^{r(\mathcal{A})-r\left(\langle S\rangle_{\mathcal{A}}\right)}
$$

Finally, define

$$
\operatorname{vol} C P_{\mathcal{A}}=\sum_{S \in \mathcal{B}(\mathcal{A})} \operatorname{vol} P_{\mathcal{A}}^{S}, \operatorname{vol} P_{\mathcal{A}}=\sum_{S \in \mathcal{I}(\mathcal{A})} \operatorname{vol} P_{\mathcal{A}}^{S},
$$

and

$$
\operatorname{vol}_{q} P_{\mathcal{A}}=\sum_{S \in \mathcal{I}(\mathcal{A})} \operatorname{vol}_{q} P_{\mathcal{A}}^{S}
$$

where $\mathcal{I}(\mathcal{M})$ denotes the independent sets of the matroid $\mathcal{M}$.

\section{Theorem 1}

$$
\operatorname{vol}_{q} P_{\mathcal{A}}=(-2 \pi)^{r(\mathcal{A})} \chi_{\mathcal{A}}(-q)
$$

where $\chi_{\mathcal{A}}(t)$ is the characteristic polynomial of the central hyperplane arrangement $\mathcal{A}$ in $\mathbb{C}^{r}$ with $\operatorname{dim}\left(\bigcap_{H \in \mathcal{A}} H\right)=0$. 
In particular,

$$
\operatorname{vol} C P_{\mathcal{A}}=(-2 \pi)^{r(\mathcal{A})} \chi_{\mathcal{A}}(0)
$$

We prove Theorem 1 in the next section.

We now point out an alternative way of thinking about the $q$-volume. Recall that $\langle S\rangle_{\mathcal{A}}$ has the structure of a matroid. Two hyperplanes $H_{k}$ and $H_{l}$ are in the same connected component of $\langle S\rangle_{\mathcal{A}}$ if they are in a common circuit of the matroid defined by $\langle S\rangle_{\mathcal{A}}$. Let the components of $\langle S\rangle_{\mathcal{A}}$ be $C_{1}, \ldots, C_{k}$. Then it is possible to write $S=\bigsqcup_{l=1}^{k} S_{l}$ such that $C_{i}=\left\langle S_{i}\right\rangle_{\mathcal{A}}$. We call $S_{1}, \ldots, S_{k}$ the components of $S$. as

The volume $\operatorname{vol}_{q} P_{\langle S\rangle_{\mathcal{A}}}^{S}$, where $S_{1}, \ldots, S_{k}$ are the components of $S$, can be written

$$
\operatorname{vol}_{q} P_{\langle S\rangle_{\mathcal{A}}}^{S}=\operatorname{vol}_{q} P_{\left\langle S_{1}\right\rangle_{\mathcal{A}}}^{S_{1}} \cdots \operatorname{vol}_{q} P_{\left\langle S_{k}\right\rangle_{\mathcal{A}}}^{S_{k}}
$$

where $S_{1}, \ldots, S_{k}$ are the components of $S$.

\section{Proof of Theorem 1}

In this section we prove Theorem 1. We first state a few lemmas we use in the proof.

Lemma 2 (Invariance Lemma) The volume $\sum_{S:|S|=s, S \in \mathcal{I}(\mathcal{A})}$ vol $P_{\mathcal{A}}^{S}$ is independent of the $R_{i}, i \in[N]$, where $s \in\{0,1, \ldots, r(\mathcal{A})\}$.

Lemma 2 is more general than the Invariance Lemma stated and proved in [3], but the techniques used in [3] also apply in this case, yielding the proof of Lemma 2. It is essential for the proof of the Invariance Lemma in [3] that the branched polymers are considered in the plane. A key observation benefiting the arguments (and calculations) in [3] is that the sum of interior angles of polygons in the plane is fixed. Note that the analogue of the Invariance Lemma is not true in 3 (real) dimensions. While our notion of branched polymers is broader than the one used by Kenyon and Winkler, our branched polymers are still points in the complex plane, and as such remain two (real) dimensional, and because of this the arguments in [3] apply. Note also that in Lemma 2, and in the rest of the paper, we consider a central hyperplane arrangement $\mathcal{A}$.

Lemma 3 Let $\mathcal{A}^{\prime}=\mathcal{A} \backslash\left\{H_{1}\right\}$ such that $r(\mathcal{A})=r\left(\mathcal{A}^{\prime}\right)$. Then as $R_{1} \rightarrow 0$,

$$
\operatorname{vol} P_{\mathcal{A}^{\prime}}^{S}=\operatorname{vol} P_{\mathcal{A}}^{S}
$$

for any independent set $S \subset \mathcal{A}$, with $H_{1} \notin S$.

Proof Note that $r\left(\langle S\rangle_{\mathcal{A}}\right)=r\left(\langle S\rangle_{\mathcal{A}^{\prime}}\right)$. Let $\left\{\left\langle S_{1}\right\rangle_{\mathcal{A}}, \ldots,\left\langle S_{k}\right\rangle_{\mathcal{A}}\right\}$ be the set of components of $\langle S\rangle_{\mathcal{A}}$ and let $\left\{\left\langle S_{1 i}\right\rangle_{\mathcal{A}^{\prime}}\right\}_{i \in I_{1}} \cup \ldots \cup\left\{\left\langle S_{k i}\right\rangle_{\mathcal{A}^{\prime}}\right\}_{i \in I_{k}}$ be the set of components of $\langle S\rangle_{\mathcal{A}^{\prime}}$. Then $\bigcup_{i \in I_{l}} S_{l i}=S_{l}, l \in[k]$, since if two elements $x$ and $y$ in the same component of $\langle S\rangle_{\mathcal{A}^{\prime}}$, then there is a circuit in $\mathcal{A}$ containing $x, y$ and not containing $H_{1}$. Also, if elements $x$ and $y$, which belong to different components of $\langle S\rangle_{\mathcal{A}^{\prime}}$, are contained in a circuit in $\mathcal{A}$ (then this circuit necessarily contains $H_{1}$ ) and if $x^{\prime}$ and $x$ are in a circuit of $\mathcal{A}$ and $x$ and $y$ are in a circuit of $\mathcal{A}$, then $x^{\prime}$ and $y$ are in a circuit of $\mathcal{A}$. 
We have

$$
\operatorname{vol} P_{\mathcal{A}}^{S}=\prod_{j=1}^{k} \operatorname{vol} P_{\left\langle S_{j}\right\rangle_{\mathcal{A}}}^{S_{j}} \cdot(2 \pi)^{r(\mathcal{A})-r\left(\langle S\rangle_{\mathcal{A}}\right)}
$$

and

$$
\operatorname{vol} P_{\mathcal{A}^{\prime}}^{S}=\prod_{j=1}^{k} \prod_{i \in I_{j}} \operatorname{vol} P_{\left\langle S_{j i}\right\rangle_{\mathcal{A}^{\prime}}}^{S_{j i}} \cdot(2 \pi)^{r\left(\mathcal{A}^{\prime}\right)-r\left(\langle S\rangle_{\mathcal{A}^{\prime}}\right)}
$$

We claim that for any $j \in[k]$,

$$
\operatorname{vol} P_{\left\langle S_{j}\right\rangle_{\mathcal{A}}}^{S_{j}}=\prod_{i \in I_{j}} \operatorname{vol} P_{\left\langle S_{j i}\right\rangle_{\mathcal{A}^{\prime}}}^{S_{j i}} .
$$

Since $r(\mathcal{A})-r\left(\langle S\rangle_{\mathcal{A}}\right)=r\left(\mathcal{A}^{\prime}\right)-r\left(\langle S\rangle_{\mathcal{A}^{\prime}}\right)$, to prove that vol $P_{\mathcal{A}}^{S}=\operatorname{vol} P_{\mathcal{A}^{\prime}}^{S}$, it suffices to establish Eq. (6).

Equation (6) follows, since, as $R_{1} \rightarrow 0$, the presence of the hyperplane $H_{1}$ in $\mathcal{A}$ imposes no constraints on the angles $\varphi_{j}$, for $j \in S_{j i}$. Furthermore, if $H_{l} \in\left\langle S_{j l}\right\rangle_{\mathcal{A}^{\prime}}$ and $H_{m} \in\left\langle S_{j m}\right\rangle_{\mathcal{A}^{\prime}}, l \neq m$, then as $R_{1} \rightarrow 0$, the span of the angles $\varphi_{l}$ and $\varphi_{m}$ are independent of each other as all circuits containing both of them also contain $H_{1}$.

Lemma 4 Let $\mathcal{A}^{\prime \prime}=\mathcal{A} /\left\{H_{1}\right\}$ be the contraction of $\mathcal{A}$ with respect to the hyperplane $H_{1}$ such that $r(\mathcal{A})=r\left(\mathcal{A}^{\prime \prime}\right)+1$. Then as $R_{1} \rightarrow 0$,

$$
2 \pi \cdot \operatorname{vol} P_{\mathcal{A}^{\prime \prime}}^{S \backslash\left\{H_{1}\right\}}=\operatorname{vol} P_{\mathcal{A}}^{S},
$$

for any independent set $S \subset \mathcal{A}$ with $H_{1} \in S$.

Proof Note that since $S$ is an independent set in $\mathcal{A}$, so is $S \backslash\left\{H_{1}\right\}$ in $\mathcal{A}^{\prime \prime}$. Also, $r\left(\langle S\rangle_{\mathcal{A}}\right)=$ $r\left(\left\langle S \backslash\left\{H_{1}\right\}\right\rangle_{\mathcal{A}^{\prime \prime}}\right)+1$ and $r(\mathcal{A})-r\left(\langle S\rangle_{\mathcal{A}}\right)=r\left(\mathcal{A}^{\prime \prime}\right)-r\left(\left\langle S \backslash\left\{H_{1}\right\}\right\rangle_{\mathcal{A}^{\prime \prime}}\right)$. Thus, it suffices to prove that $2 \pi \cdot \operatorname{vol} P_{\left\langle S \backslash\left\{H_{1}\right\}\right\rangle_{\mathcal{A}^{\prime \prime}}}^{S \backslash\left\{H_{1}\right\}}=\operatorname{vol} P_{\langle S\rangle_{\mathcal{A}}}^{S}$.

Let the set of components of $\langle S\rangle_{\mathcal{A}}$ be $\left\{\left\langle S_{1}\right\rangle_{\mathcal{A}}, \ldots,\left\langle S_{k}\right\rangle_{\mathcal{A}}\right\}$ with $H_{1} \in S_{1}$. Note that any circuit in $\langle S\rangle_{\mathcal{A}}$ not involving $H_{1}$ automatically carries over to a circuit in $\langle S\rangle_{\mathcal{A}^{\prime \prime}}$ (easy to see if we assume, without loss of generality, that $h_{1}(\mathbf{x})=x_{n}$ ). Also, any circuit in $\langle S\rangle_{\mathcal{A}^{\prime \prime}}$ automatically lifts to a circuit in $\langle S\rangle_{\mathcal{A}}$. Thus, the set of components of $\left\langle S \backslash\left\{H_{1}\right\}\right\rangle_{\mathcal{A}^{\prime \prime}}$ is $\left\{\left\langle S_{1 j}\right\rangle_{\mathcal{A}^{\prime \prime}}\right\}_{j \in I_{1}} \cup\left\{\left\langle S_{2}\right\rangle_{\mathcal{A}^{\prime \prime}}, \ldots,\left\langle S_{k}\right\rangle_{\mathcal{A}^{\prime \prime}}\right\}$, where $\bigcup_{j \in I_{1}} S_{1 j}=S_{1}$. As $R_{1} \rightarrow 0$, the volumes vol $P_{\left\langle S_{i}\right\rangle_{\mathcal{A}^{\prime \prime}}}^{S_{i}}=\operatorname{vol} P_{\left\langle S_{i}\right\rangle_{\mathcal{A}}}^{S_{i}}, i \in\{2, \ldots, k\}$. Finally, if $H_{l} \in\left\langle S_{1 l}\right\rangle_{\mathcal{A}}$ and $H_{m} \in\left\langle S_{1 m}\right\rangle_{\mathcal{A}}, l \neq m$, then as $R_{1} \rightarrow 0$, the span of the angles $\varphi_{l}$ and $\varphi_{m}$ are independent of each other as all circuits containing both of them also contain $H_{1}$. Thus, $2 \pi \cdot \prod_{j \in I_{1}} \operatorname{vol} P_{\left\langle S_{1 j}\right\rangle_{\mathcal{A}^{\prime \prime}}}^{S_{1} j}=\operatorname{vol} P_{\left\langle S_{1}\right\rangle_{\mathcal{A}}}^{S_{1}}$, the $2 \pi$ accounting for the fact that $H_{1} \in S_{1}$ and as $R_{1} \rightarrow 0, \varphi_{1}$ ranges between 0 and $2 \pi$ freely. 
Proof of Theorem 1 Equation (5) is a consequence of (4) by the definitions of the spaces $P_{\mathcal{A}}$ and $C P_{\mathcal{A}}$ and their notions of volume. To prove Eq. (4) we consider two cases depending on whether there is a hyperplane $H \in \mathcal{A}$ such that $r(\mathcal{A})=r\left(\mathcal{A}^{\prime}\right)$, for $\mathcal{A}^{\prime}=\mathcal{A} \backslash\{H\}$.

Suppose $\mathcal{A}$ is such that if we delete any hyperplane $H$ from it, obtaining the hyperplane arrangement $\mathcal{A}^{\prime}=\mathcal{A} \backslash\{H\}$, then $r\left(\mathcal{A}^{\prime}\right)=r(\mathcal{A})-1$. This means that the hyperplanes of $\mathcal{A}$ constitute a base. Then

$$
\begin{aligned}
\operatorname{vol}_{q} P_{\mathcal{A}} & =\sum_{S: S \in \mathcal{I}(\mathcal{A})} q^{r(\mathcal{A})-|S|} \operatorname{vol} P_{\mathcal{A}}^{S} \\
& =\sum_{i=0}^{r(\mathcal{A})}\left(\begin{array}{c}
r(\mathcal{A}) \\
i
\end{array}\right) q^{r(\mathcal{A})-i}(2 \pi)^{r(\mathcal{A})} \\
& =(1+q)^{r(\mathcal{A})}(2 \pi)^{r(\mathcal{A})} \\
& =(-2 \pi)^{r(\mathcal{A})}(-q-1)^{r(\mathcal{A})} \\
& =(-2 \pi)^{r(\mathcal{A})} \chi_{\mathcal{A}}(-q) .
\end{aligned}
$$

The second equality holds since the independent sets of size $i$ are exactly the $i$-subset of the base $\mathcal{A}$, of which there are $\left(\begin{array}{c}r(\mathcal{A}) \\ i\end{array}\right)$ and vol $P_{\mathcal{A}}^{S}=(2 \pi)^{r(\mathcal{A})}$ for any set $S$, since $\mathcal{A}$ is a base itself. The last equality holds since if $\mathcal{A}$ itself is a base, then $\chi_{\mathcal{A}}(t)=(t-1)^{r(\mathcal{A})}$.

Suppose now that there exists a hyperplane $H \in \mathcal{A}$ such that $r(\mathcal{A})=r\left(\mathcal{A}^{\prime}\right)$ for $\mathcal{A}^{\prime}=\mathcal{A} \backslash\{H\}$. Assume without loss of generality that $H=H_{1}$. If $\mathcal{A}^{\prime}$ and $\mathcal{A}^{\prime \prime}$ are the deletion and restriction of $\mathcal{A}$ with respect to the hyperplane $h_{1}(\mathbf{x})=0$, then $r(\mathcal{A})=r\left(\mathcal{A}^{\prime}\right)=r\left(\mathcal{A}^{\prime \prime}\right)+1$. Recall that

$$
\chi_{\mathcal{A}}(t)=\chi_{\mathcal{A}^{\prime}}(t)-\chi_{\mathcal{A}^{\prime \prime}}(t)
$$

Let

$$
R(\mathcal{A}, q)=(-2 \pi)^{r(\mathcal{A})} \chi_{\mathcal{A}}(-q)
$$

denote the right hand side of Eq. (4). By (7)

$$
R(\mathcal{A}, q)=R\left(\mathcal{A}^{\prime}, q\right)+2 \pi R\left(\mathcal{A}^{\prime \prime}, q\right)
$$

In particular,

$$
\left[q^{p}\right] R(\mathcal{A}, q)=\left[q^{p}\right] R\left(\mathcal{A}^{\prime}, q\right)+2 \pi\left[q^{p}\right] R\left(\mathcal{A}^{\prime \prime}, q\right)
$$

for any integer power $p$ of $q$.

To prove the theorem it suffices to show that the same recurrence holds for

$$
L(\mathcal{A}, q)=\sum_{S: S \in \mathcal{I}(\mathcal{A})} q^{r(\mathcal{A})-|S|} \operatorname{vol} P_{\mathcal{A}}^{S},
$$


since the case when we cannot use the recurrence is exactly when removing any hyperplane from $\mathcal{A}$ reduces the rank, and we proved the validity of Theorem 1 for it above.

Recurrence (8) holds, since

$$
\begin{aligned}
\sum_{S:|S|=s, S \in \mathcal{I}(\mathcal{A})} \operatorname{vol} P_{\mathcal{A}}^{S}= & \sum_{S^{\prime}:\left|S^{\prime}\right|=s, S^{\prime} \in \mathcal{I}\left(\mathcal{A}^{\prime}\right)} \operatorname{vol} P_{\mathcal{A}^{\prime}}^{S^{\prime}} \\
& +2 \pi \sum_{S^{\prime \prime}:\left|S^{\prime \prime}\right|=s-1, S^{\prime \prime} \in \mathcal{I}\left(\mathcal{A}^{\prime \prime}\right)} \operatorname{vol} P_{\mathcal{A}^{\prime \prime}}^{S^{\prime \prime}},
\end{aligned}
$$

since Lemmas 3 and 4 imply

$$
\sum_{S^{\prime}:\left|S^{\prime}\right|=s, S^{\prime} \in \mathcal{I}\left(\mathcal{A}^{\prime}\right)} \operatorname{vol} P_{\mathcal{A}^{\prime}}^{S^{\prime}}=\sum_{S: H_{1} \notin S,|S|=s, S \in \mathcal{I}(\mathcal{A})} \operatorname{vol} P_{\mathcal{A}}^{S}
$$

and

$$
2 \pi \sum_{S^{\prime \prime}:\left|S^{\prime \prime}\right|=S-1, S^{\prime \prime} \in \mathcal{I}\left(\mathcal{A}^{\prime \prime}\right)} \operatorname{vol} P_{\mathcal{A}^{\prime \prime}}^{S^{\prime \prime}}=\sum_{S: H_{1} \in S,|S|=S, S \in \mathcal{I}(\mathcal{A})} \operatorname{vol} P_{\mathcal{A}}^{S} .
$$

Example By Theorem 1 the $q$-volume of the space of branched polymers associated to the braid arrangement is

$$
\begin{aligned}
\operatorname{vol}_{q} P_{\mathcal{B}_{n}} & =(-2 \pi)^{r\left(\mathcal{B}_{n}\right)} \chi_{\mathcal{B}_{n}}(-q) \\
& =(2 \pi)^{n-1}(q+1) \cdots(q+(n-1)) .
\end{aligned}
$$

A special case of (11) is Eq. (1).

\section{G-Polymers}

In this section we discuss $G$-polymers, as defined in [3, Sect. 3], and show how they correspond to graphical arrangements in our setup. We also rewrite the $q$-volume of the generalized $G$-polymers in the language of its chromatic polynomial, which is a special case of its Tutte polynomial.

Given a graph $G=([n], E)$, let $R_{i j}$ be positive scalars for each edge $(i, j)$ of $G$. A $G$-polymer, following Kenyon and Winkler, is a configuration of points $\left(x_{1}, \ldots, x_{n}\right) \in$ $\mathbb{C}^{n}$ such that

- $\left|x_{i}-x_{j}\right| \geq R_{i j}$, for any edge $(i, j)$ of $G$

- $\left|x_{i}-x_{j}\right|=R_{i j}$ for all edges $(i, j)$ of some connected subgraph of $G$.

If $G$ is not connected, there are no $G$-polymers. The volume of the space of $G$-polymers is defined by the angles made by the vectors from $x_{i}$ to $x_{j}$, where $(i, j) \in E$ is such that $\left|x_{i}-x_{j}\right|=R_{i j}$. See [3] for further details. 
Recall that the graphical arrangement $\mathcal{A}_{G}$ corresponding to the graph $G=([n], E)$ consists of the $|E|$ hyperplanes

$$
H_{i j}=\left\{\mathbf{x} \in \mathbb{C}^{n} \mid x_{i}-x_{j}=0\right\} \text { for }(i, j) \in E, i<j .
$$

We consider $\mathcal{A}_{G}$ as a hyperplane arrangement in $\mathbb{C}^{n} /\left(\bigcap_{(i, j) \in E} H_{i j}\right)$. The space of connected branched polymers associated to $\mathcal{A}_{G}$ as defined in (3) coincide with the space of $G$-polymers.

Let $\chi_{G}(t)$ be the chromatic polynomial of the graph $G, \tilde{\chi}_{G}(t)=\chi_{G}(t) / t^{k(G)}$, where $k(G)$ is the number of components of $G$ and $r(G)=n-k(G)$. A special case of Theorem 1 is the following proposition.

Proposition 5 ([3, Theorem 4])

$$
\operatorname{vol} C P_{\mathcal{A}_{G}}=(-2 \pi)^{r(G)} \tilde{\chi}_{G}(0)
$$

Proposition 5 follows from Theorem 1 since $\tilde{\chi}_{G}(t)=\chi_{\mathcal{A}_{G}}(t)[5$, Theorem 2.7] and $r(G)=r\left(\mathcal{A}_{G}\right)$.

Recall also that the chromatic polynomial $\chi_{G}(t)$ of a graph $G$ is a special case of its Tutte polynomial $T_{G}(x, y): \chi_{G}(t)=(-1)^{r(G)} t^{k(G)} T_{G}(1-t, 0)$ [1, Theorem 6 , p. 346]. In this light, Proposition 5 coincides with [3, Theorem 4], which is stated in terms of the Tutte polynomial of $G$.

The space of generalized $G$-polymers is $P_{\mathcal{A}_{G}}$ as defined in (2). A special case of Theorem 1 is the following proposition.

\section{Proposition 6}

$$
\operatorname{vol}_{q} P_{\mathcal{A}_{G}}=(-2 \pi)^{r(G)} \tilde{\chi}_{G}(-q) .
$$

As Proposition 6 illustrates, the notion of $q$-volume of the space of generalized $G$-polymers allows for recovering the entire chromatic polynomial of $G$.

\section{Symmetric Polymers}

In this section we specialize our results to the type $B_{n}$ Coxeter arrangement. This arrangement naturally gives rise to polymers symmetric with respect to the origin.

The type $B_{n}$ Coxeter arrangement $\mathcal{A}\left(B_{n}\right), n>1$, in $\mathbb{C}^{n}$, consists of the $2\left(\begin{array}{l}n \\ 2\end{array}\right)+n$ hyperplanes

$$
\begin{aligned}
H_{i j}^{-} & =\left\{\mathbf{x} \in \mathbb{C}^{n} \mid x_{i}-x_{j}=0\right\}, H_{i j}^{+}=\left\{\mathbf{x} \in \mathbb{C}^{n} \mid x_{i}+x_{j}=0\right\}, \\
H_{k} & =\left\{\mathbf{x} \in \mathbb{C}^{n} \mid x_{k}=0\right\}, \text { for } 1 \leq i<j \leq n, k \in[n] .
\end{aligned}
$$

The space of branched polymers associated to $\mathcal{A}\left(B_{n}\right)$ and positive scalars $R_{i j}^{+}, R_{i j}^{-}, R_{k}$, for $1 \leq i<j \leq n, k \in[n]$ is, by definition, 


$$
\begin{aligned}
P_{\mathcal{A}\left(B_{n}\right)}= & \left\{\mathbf{x} \in V|| x_{i}+x_{j}\left|\geq R_{i j}^{+},\right| x_{i}-x_{j}\left|\geq R_{i j}^{-},\right| x_{k} \mid \geq R_{k},\right. \\
& \text { for } 1 \leq i<j \leq n, k \in[n]\} .
\end{aligned}
$$

To obtain symmetric polymers, place a disk of radius $r_{k}$ around the points $x_{k}$ and $-x_{k}$ in the plane for all $k \in[n]$. Let $R_{i j}^{+}=R_{i j}^{-}=r_{i}+r_{j}$ and $R_{k}=r_{k}$, for $1 \leq i<j \leq n, k \in[n]$. Then the condition $\left|x_{i}+x_{j}\right| \geq R_{i j}^{+}$ensures that the disks around $x_{i}$ and $-x_{j}$, and $-x_{i}$ and $x_{j}$ do not overlap, the condition $\left|x_{i}-x_{j}\right| \geq R_{i j}^{-}$ ensures that the disks around $x_{i}$ and $x_{j}$, and $-x_{i}$ and $-x_{j}$ do not overlap, and the condition $\left|x_{k}\right| \geq R_{k}$ ensures that the disks around $x_{k}$ and $-x_{k}$ do not overlap.

Since the characteristic polynomial of $\mathcal{A}\left(B_{n}\right)$ is given by

$$
\chi_{\mathcal{A}\left(B_{n}\right)}(t)=(t-1) \cdot(t-3) \cdots(t-(2 n-1)),[5, p .451]
$$

Theorem 1 specializes to the following corollary.

\section{Corollary 7}

$$
\operatorname{vol}_{q} P_{\mathcal{A}\left(B_{n}\right)}=(2 \pi)^{n}(q+1) \cdot(q+3) \cdots(q+(2 n-1)) .
$$

\section{In particular,}

$$
\operatorname{vol} C P_{\mathcal{A}\left(B_{n}\right)}=(2 \pi)^{n} 1 \cdot 3 \cdots(2 n-1) \text {. }
$$

We now outline the geometric picture of connected branched polymers associated to $\mathcal{A}\left(B_{n}\right)$. Since vol $C P_{\mathcal{A}\left(B_{n}\right)}=\sum_{S \in \mathcal{B}\left(\mathcal{A}\left(B_{n}\right)\right)}$ vol $P_{\mathcal{A}\left(B_{n}\right)}^{S}$ we restrict our attention to the polymers corresponding to the bases of $\mathcal{A}\left(B_{n}\right)$. Biject a subset $S$ of hyperplanes of $\mathcal{A}\left(B_{n}\right)$ with a graph $G_{S}$ on vertex set $[n]$ by adding an edge labeled by + between vertices $i$ and $j$ if $H_{i j}^{+} \in S$, by adding an edge labeled by - between vertices $i$ and $j$ if $H_{i j}^{-} \in S$, and adding a loop at vertex $k$ if $H_{k} \in S$. Then the bases of $\mathcal{A}\left(B_{n}\right)$ correspond to graphs $G$ on vertex set $[n]$ with $n$ edges such that each component of $G$ contains exactly one cycle or one loop, and if it contains a cycle then the cycle has an odd number of edges labeled by + .

It is easy to see that if there is more than one loop in $G_{S}$, for some base $S$, then the stratum $P_{\mathcal{A}\left(B_{n}\right)}^{S}$ is empty. This is true, since a loop based at vertex $k$ in $G_{S}$ corresponds to two disks of radius $R_{k}=r_{k}$ around the points $x_{k}$ and $-x_{k}$, touching at the origin. If there was also a loop at vertex $l \neq k$, it would be impossible to also satisfy $\left|x_{k}+x_{l}\right| \geq$ $r_{l}+r_{k}$ and $\left|x_{k}-x_{l}\right| \geq r_{l}+r_{k}$ if $k<l$ or $\left|x_{l}-x_{k}\right| \geq r_{l}+r_{k}$ if $k>l$. The disks corresponding to a component of $G_{S}$ with a loop at $k$ look like a tree symmetric across the origin and can be parametrized by the angles which the edges of "half" of the tree make with the $x$-axis and by the angle made by the segment $0-x_{k}$ and the $x$-axis; see Fig. 2. The disks corresponding to a component of $G_{S}$ with a cycle is symmetric with respect to the origin and contains a cycle of disks symmetrically around the origin. If the cycle is of length $2 m$, then the polymer is parametrized by $m+1$ angles which the edges of the cycle make with the $x$-axis as well the angles that "half" of the remaining edges make with the $x$-axis; see Fig. 3 . 
Fig. 2 The graph representing a base and the corresponding polymer. The half-line drawn on the figure is the positive $x$-axis. The parametrization can be done by the angles which the dashed line segments make with the $x$-axis

Fig. 3 The graph representing a base and the corresponding polymer. The half-line drawn on the figure is the positive $x$-axis. The parametrization can be done by the angles which the dashed line segments make with the $x$-axis
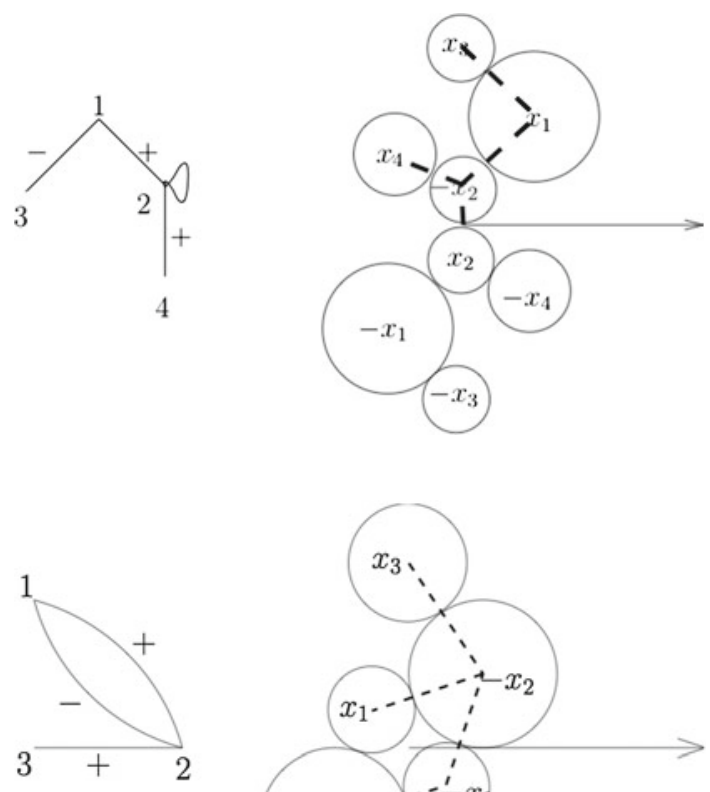

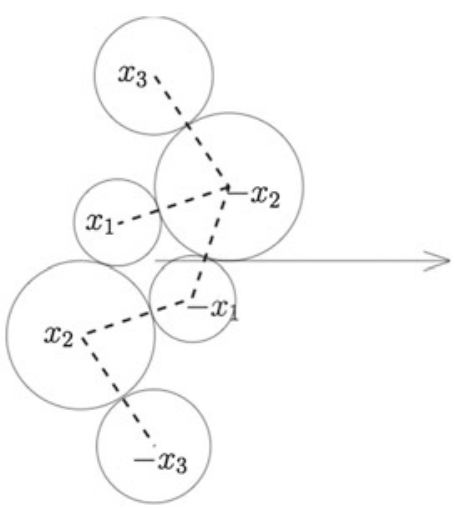

\section{Volumes of Branched Polymers and Broken Circuits}

In this section we relate the volumes of branched polymers associated to $\mathcal{A}$ and broken circuits of $\mathcal{A}$. While the volume vol $C P_{\mathcal{A}}$ is invariant under changing the radii $R_{i}$ by the Invariance Lemma, the individual volumes of the strata $P_{\mathcal{A}}^{S}$ change. By picking the radii $R_{i}$ appropriately, it is possible to construct a stratification, where only strata corresponding to certain special bases $S$ appear in the stratification, and they all have the same volume. The mentioned special bases are the no broken circuit bases as we show in Theorem 8.

A broken circuit of $\mathcal{A}$ with respect to a linear ordering $\mathcal{O}$ of the hyperplanes of $\mathcal{A}$ is a set $C-\{u\}$, where $C$ is a circuit and $u$ is the largest element of $C$ in the linear ordering $\mathcal{O}$. While the exact set of broken circuits depends on the linear order we choose, remarkably, the number of sets $S$ of hyperplanes of size $k$, for an arbitrary fixed $k$, such that $S$ contains no broken circuit is the same; see [5, Theorem 4.12].

Theorem 8 Let $\mathcal{A}=\left\{H_{1}, \ldots, H_{N}\right\}$ be a hyperplane arrangement with $R_{1} \ll \cdots \ll$ $R_{N}$. Then the space $C P_{\mathcal{A}}$ is a disjoint union of $(-1)^{r(\mathcal{A})} \chi_{\mathcal{A}}(0)$ tori $\left(S^{1}\right)^{r(\mathcal{A})}$.

More precisely, if $\mathcal{O}$ is the order $H_{1}<\cdots<H_{N}$ on the hyperplanes of $\mathcal{A}$ and $B C_{\mathcal{O}}(\mathcal{A})$ are all subsets of $\mathcal{A}$ containing no broken circuits with respect to $\mathcal{O}$, then for $R_{1} \ll \cdots \ll R_{N}$ 


$$
C P_{\mathcal{A}}=\bigsqcup_{S \in \mathcal{B}(\mathcal{A}) \cap B C_{\mathcal{O}}(\mathcal{A})} P_{\mathcal{A}}^{S}
$$

where vol $P_{\mathcal{A}}^{S}=(2 \pi)^{r(\mathcal{A})}$ for $S \in \mathcal{B}(\mathcal{A}) \cap B C_{\mathcal{O}}(\mathcal{A})$.

Proof By [5, Theorem 4.12]

$$
\#\left\{S|| S \mid=i, S \in B C_{\mathcal{O}}(\mathcal{A})\right\}=(-1)^{r(\mathcal{A})}\left[q^{r(\mathcal{A})-i)}\right] \chi_{\mathcal{A}}(-q)
$$

Thus, $\left|\mathcal{B}(\mathcal{A}) \cap B C_{\mathcal{O}}(\mathcal{A})\right|=(-1)^{r(\mathcal{A})} \chi_{\mathcal{A}}(0)$, and it suffices to prove the second part of the theorem's statement.

Recall that by definition

$$
C P_{\mathcal{A}}=\bigsqcup_{S \subset \mathcal{A}: r(S)=r(\mathcal{A})} P_{\mathcal{A}}^{S}
$$

We first show that if $S$ contains a broken circuit, then $P_{\mathcal{A}}^{S}$ is empty. Let $\left\{H_{i_{1}}, \ldots, H_{i_{k}}\right\} \subset S, i_{1}<\cdots<i_{k}$, be a broken circuit. Then there exists $H_{i} \in \mathcal{A}$ such that $\left\{H_{i_{1}}, \ldots, H_{i_{k}}, H_{i}\right\}$ is a circuit and $i_{k}<i$. Let $h_{i}=\sum_{j=1}^{k} c_{j} h_{i_{j}}, c_{j} \neq 0$. Then for any $\mathbf{x} \in P_{\mathcal{A}}^{S_{j}}$,

$$
\sum_{j=1}^{k}\left|c_{j}\right| R_{i_{k}} \geq \sum_{j=1}^{k}\left|c_{j}\right| R_{i_{j}}=\sum_{j=1}^{k}\left|c_{j} h_{i_{j}}(\mathbf{x})\right| \geq\left|\sum_{j=1}^{k} c_{j} h_{i_{j}}(\mathbf{x})\right|=\left|h_{i}(\mathbf{x})\right| \geq R_{i}
$$

However,

$$
\sum_{j=1}^{k}\left|c_{j}\right| R_{i_{k}}<R_{i}, \text { since } R_{i_{k}} \ll R_{i}
$$

Thus, $P_{\mathcal{A}}^{S}$ is empty.

Next we prove that $\operatorname{vol} P_{\mathcal{A}}^{S}=(2 \pi)^{r(\mathcal{A})}$ for $S \in \mathcal{B}(\mathcal{A}) \cap B C_{\mathcal{O}}(\mathcal{A})$. Let $S=$ $\left\{H_{j_{1}}, \ldots, H_{j_{r}}\right\}$ with $j_{1}<\cdots<j_{r}$. Since $S$ contains no broken circuit, it follows that if $i \in[N] \backslash\left\{j_{1}, \ldots, j_{r}\right\}$, then $i<j_{r}$. To prove that $\operatorname{vol}_{\mathcal{A}}^{S}=(2 \pi)^{r(\mathcal{A})}$, it suffices to show that $\left|h_{j_{l}}(\mathbf{x})\right|=R_{j_{l}}$ for $l \in[r]$ imply $\left|h_{i}(\mathbf{x})\right|>R_{i}$ for $i \in[N] \backslash\left\{j_{1}, \ldots, j_{r}\right\}$. Since $S$ is a base, $S \cup\left\{H_{i}\right\}$ contains a circuit $C$ whose maximal element is $H_{j_{k}} \neq H_{i}, k \in[r]$, since $S \in B C_{\mathcal{O}}(\mathcal{A})$. Thus,

$$
h_{j_{k}}(\mathbf{x})=c_{i} h_{i}(\mathbf{x})+\sum_{l \in[N] \backslash\{k\}} c_{j_{l}} h_{j_{l}}(\mathbf{x}),
$$


where $c_{i}, c_{j_{1}}, \ldots, c_{j_{r}}$ are scalars with $c_{i} \neq 0$. In particular,

$$
\left|h_{j_{k}}(\mathbf{x})\right| \leq\left|c_{i}\right|\left|h_{i}(\mathbf{x})\right|+\sum_{l \in[N] \backslash\{k\}}\left|c_{j_{l}}\right|\left|h_{j_{l}}(\mathbf{x})\right| .
$$

Since $\left|h_{j_{l}}(\mathbf{x})\right|=R_{j_{l}}$ for $l \in[r]$ and $R_{j_{k}} \gg R_{i}$ it follows that $\left|h_{i}(\mathbf{x})\right|>R_{i}$.

An alternative proof of Theorem 1 By definition,

$$
\operatorname{vol}_{q} P_{\mathcal{A}}=\sum_{i=0}^{r(\mathcal{A})} \sum_{S \in \mathcal{S}_{i}}(q \cdot 2 \pi)^{r(\mathcal{A})-i} \operatorname{vol} C P_{\langle S\rangle_{\mathcal{A}}}
$$

where $\mathcal{S}_{i}$ is a collection of independent sets $S$ of cardinality $i$ such that the arrangements $\langle S\rangle_{\mathcal{A}}$ for $S \in \mathcal{S}_{i}$ run over all rank $i$ subarrangements of $\mathcal{A}$. By Theorem 8 the right hand side of Eq. (17) is

$$
\begin{array}{r}
\sum_{i=0}^{r(\mathcal{A})} \sum_{S \in \mathcal{S}_{i}}(q \cdot 2 \pi)^{r(\mathcal{A})-i} \#\left\{B \mid B \in \mathcal{B}\left(\langle S\rangle_{\mathcal{A}}\right) \cap B C_{\mathcal{O}}\left(\langle S\rangle_{\mathcal{A}}\right)\right\} \cdot(2 \pi)^{i} \\
=\sum_{i=0}^{r(\mathcal{A})} q^{r(\mathcal{A})-i} \cdot(2 \pi)^{r(\mathcal{A})} \#\left\{S|| S \mid=i, S \in B C_{\mathcal{O}}(\mathcal{A})\right\} .
\end{array}
$$

Since by [5, Theorem 4.12]

$$
\#\left\{S|| S \mid=i, S \in B C_{\mathcal{O}}(\mathcal{A})\right\}=(-1)^{r(\mathcal{A})}\left[q^{r(\mathcal{A})-i)}\right] \chi_{\mathcal{A}}(-q),
$$

it follows from Eqs. (17)-(20) that

$$
\operatorname{vol}_{q} P_{\mathcal{A}}=(-2 \pi)^{r(\mathcal{A})} \chi_{\mathcal{A}}(-q)
$$

In particular,

$$
\operatorname{vol} C P_{\mathcal{A}}=(-2 \pi)^{r(\mathcal{A})} \chi_{\mathcal{A}}(0)
$$

Note the striking similarity between the formula for the $1 / t$-volume of branched polymers $P_{\mathcal{A}}$,

$$
\operatorname{vol}_{1 / t} P_{\mathcal{A}}=(-2 \pi)^{r(\mathcal{A})} \chi_{\mathcal{A}}(-1 / t)
$$

and the generating function

$$
\sum_{i \geq 0} \operatorname{rank} H^{i}\left(C_{\mathcal{A}}, \mathbb{Z}\right) t^{i}=(-t)^{r(\mathcal{A})} \chi_{\mathcal{A}}(-1 / t),
$$


where $C_{\mathcal{A}}=\mathbb{C}^{r} \backslash \bigcup_{H \in \mathcal{A}} H$, is the complement of the arrangement $\mathcal{A}$ in $\mathbb{C}^{r}$ and $H^{k}\left(C_{\mathcal{A}}, \mathbb{Z}\right)$ is the $k$ th graded piece of the cohomology ring $H^{*}\left(C_{\mathcal{A}}, \mathbb{Z}\right)$; see [4]. The right hand sides of (21) and (22) are in fact identical, if we normalize appropriately. This is no surprise, since we related $\operatorname{vol}_{1 / t} P_{\mathcal{A}}$ to no broken circuits in Theorem 8 , and since the cohomology ring $H^{*}\left(C_{\mathcal{A}}, \mathbb{Z}\right)$ of $C_{\mathcal{A}}$ is isomorphic to the Orlik-Solomon algebra $A(\mathcal{A})$ associated to the hyperplane arrangement $\mathcal{A}$, and the $i$ th graded piece of $A(\mathcal{A})$ has a basis in terms of no broken circuits.

\section{Cohomology Rings of the Spaces $P_{\mathcal{A}}$ and $C P_{\mathcal{A}}$ and the Orlik-Solomon Algebra}

In this section we discuss the cohomology rings of the spaces $P_{\mathcal{A}}$ and $C P_{\mathcal{A}}$. The wellknown Orlik-Solomon algebra is isomorphic to the cohomology ring $H^{*}\left(C_{\mathcal{A}}, \mathbb{Z}\right)$ of $C_{\mathcal{A}}$, where $C_{\mathcal{A}}=\mathbb{C}^{r} \backslash \bigcup_{H \in \mathcal{A}} H$ is the complement of the arrangement $\mathcal{A}$ in $\mathbb{C}^{r}$. We prove that the spaces $P_{\mathcal{A}}$ and $C_{\mathcal{A}}$ are homotopy equivalent.

Proposition 9 The map $f: C_{\mathcal{A}} \rightarrow P_{\mathcal{A}}$ defined by

$$
f:\left(x_{1}, \ldots, x_{r}\right) \mapsto \max _{i \in[r]}\left(1, \frac{R_{i}}{\left|h_{i}(\mathbf{x})\right|}\right) \cdot\left(x_{1}, \ldots, x_{r}\right),
$$

where $\mathcal{A}$ is a hyperplane arrangements in $\mathbb{C}^{r}$,

$$
\begin{aligned}
& C_{\mathcal{A}}=\mathbb{C}^{r} \backslash \bigcup_{H \in \mathcal{A}} H, \\
& P_{\mathcal{A}}=\left\{\mathbf{x} \in \mathbb{C}^{r}|| h_{i}(\mathbf{x}) \mid \geq R_{i}\right\}
\end{aligned}
$$

is a deformation retraction.

Proof It is straightforward to check that $f$ satisfies the following three conditions:

(i) continuous,

(ii) $\left.f\right|_{P_{\mathcal{A}}}=i d$,

(iii) $f$ is homotopic to the identity.

Corollary 10 The spaces $P_{\mathcal{A}}$ and $C_{\mathcal{A}}$ are homotopy equivalent. In particular, the cohomology rings of the spaces $C_{\mathcal{A}}$ and $P_{\mathcal{A}}$, where $\mathcal{A}$ is a hyperplane arrangements in $\mathbb{C}^{r}$, satisfy

$$
H^{*}\left(P_{\mathcal{A}}, \mathbb{Z}\right) \cong H^{*}\left(C_{\mathcal{A}}, \mathbb{Z}\right)
$$

It would be interesting to understand the cohomology ring of the space $C P_{\mathcal{A}}$ and how it depends on the radii $R_{i}, i \in[N]$. In Theorem 8 we proved that for $\mathcal{A}=$ $\left\{H_{1}, \ldots, H_{N}\right\}$ and $R_{1} \ll \cdots \ll R_{N}$ the space $C P_{\mathcal{A}}$ is a disjoint union of $\left|\chi_{\mathcal{A}}(0)\right|$ tori $\left(S^{1}\right)^{r(\mathcal{A})}$. In particular, $C P_{\mathcal{A}}$ is disconnected and cannot be homotopy equivalent to $C_{\mathcal{A}}$. 
Acknowledgments Mészáros is partially supported by a National Science Foundation Postdoctoral Research Fellowship DMS 1103933. Postnikov is partially supported by NSF Grant DMS 6923772 and by NSF CAREER Award DMS 0504629.

\section{References}

1. Bollobás, B.: Modern Graph Theory. Springer, New York (1998)

2. Brydges, D.C., Imbrie, J.Z.: Branched polymers and dimension reduction. Ann. Math. 158, 1019-1039 (2003)

3. Kenyon, R., Winkler, P.: Branched polymers. Am. Math. Mon. 116(7), 612-628 (2009)

4. Orlik, P., Solomon, L.: Combinatorics and topology of complements of hyperplanes. Invent. Math. 56, 167-189 (1980)

5. Stanley, R.: An introduction to hyperplane arrangements. In: Miller, E., Reiner, V., Sturmfels, B. (eds.) Geometric Combinatorics, IAS/Park City Mathematics Series, vol. 13, pp. 389-496. American Mathematical Society, Providence, RI (2007) 